\title{
The value of yttrium-90 PET/CT after hepatic radioembolization: a pictorial essay
}

\author{
Ahmed A. Alsultan ${ }^{1}$ ( ) Maarten L. J. Smits ${ }^{1} \cdot$ Maarten W. Barentsz $^{1} \cdot$ Arthur J. A. T. Braat $^{1} \cdot$ Marnix G. E. H. Lam $^{1}$
}

Received: 16 May 2019 / Accepted: 6 July 2019 / Published online: 19 July 2019

(c) The Author(s) 2019

\begin{abstract}
Introduction Distribution of microspheres after radioembolization can be accurately visualized using PET/CT. In this pictorial essay, we aim to demonstrate the value of ${ }^{90} \mathrm{Y}$-PET/CT after radioembolization.

Methods ${ }^{90}$ Y-PET/CT imaging was routinely performed after radioembolization at our institute. Patients were scanned the same day or the day after treatment, using a scanner with time-of-flight technology. We retrospectively reviewed all ${ }^{90}$ Y-PET/ CTs from patients treated with radioembolization (both glass and resin microspheres) between January 2011 and January 2019. Five cases were selected that are illustrative of the added value of PET/CT after radioembolization.

Results ${ }^{90} \mathrm{Y}$-PET/CT allows for distribution assessment and dosimetry of ${ }^{90} \mathrm{Y}$-microspheres. It was used for the assessment of treatment success by visualization of tumor targeting, quantification of the absorbed dose, prediction of complications such as radioembolization-induced liver disease, and determining the required dosage for retreatment.

Conclusion PET/CT is an excellent modality for post-treatment imaging of ${ }^{90} \mathrm{Y}$-microspheres and could lead to improved dose planning and more personalized treatment.
\end{abstract}

Keywords Intra-arterial radioembolization $\cdot{ }^{90} \mathrm{Y}$-microspheres $\cdot$ Post-treatment imaging $\cdot{ }^{90} \mathrm{Y}$-PET/CT

\section{Background}

Radioembolization is an increasingly used locoregional treatment for hepatic tumors [1]. Glass (TheraSphere ${ }^{\circledR}$, BTG) or resin (SIR-Spheres ${ }^{\circledR}$, Sirtex) yttrium-90 $\left({ }^{90} \mathrm{Y}\right)$ microspheres are infused intra-arterially with the aim to deliver high radiation absorbed doses to tumors while sparing the surrounding healthy liver tissue. The safety of this treatment is contingent upon the distribution of microspheres within the liver and outside of the liver. An intolerable absorbed dose in the healthy liver tissue may lead to radioembolization-induced liver disease [2]. Furthermore, blood flow dynamics and variations in vascular anatomy may lead to deposition of microspheres outside of the liver (e.g., stomach, duodenum, or the lungs through arteriovenous shunting), causing potentially life-threatening complications.

The isotope ${ }^{90} \mathrm{Y}$ predominantly emits beta-radiation, but also emits a positron in a miniscule percentage $(0.0032 \%)$ of

Ahmed A. Alsultan

A.A.N.Alsultan@umcutrecht.nl

1 Department of Radiology and Nuclear Medicine, University Medical Center Utrecht, Utrecht, The Netherlands its decays. The $511-\mathrm{keV}$ photons originating from these positrons can be imaged using PET/CT scanners with time-offlight technology, and the in vivo distribution of ${ }^{90} \mathrm{Y}$-microspheres can be visualized. ${ }^{90} \mathrm{Y}$-PET/CT offers superior spatial resolution compared to ${ }^{90} \mathrm{Y}$-bremsstrahlung SPECT/ CT, and allows for more precise voxel-based dosimetry [3].

All patients treated with ${ }^{90} \mathrm{Y}$-radioembolization at our institute undergo post-treatment ${ }^{90} \mathrm{Y}$-PET/CT as part of the treatment protocol. We treated over 400 patients with ${ }^{90}$ Y-radioembolization, out of whom we selected 5 cases to illustrate the benefit of performing post-treatment ${ }^{90} \mathrm{Y}$-PET/ CT.

\section{${ }^{90} \mathrm{Y}$-radioembolization treatment workflow}

In advance of radioembolization, patients receive a work-up procedure to assess intrahepatic distribution and potential extrahepatic deposition of activity or excessive lung shunting. Technetium-99 m macroaggregated albumin $\left({ }^{99 \mathrm{~m}} \mathrm{Tc}-\right.$ MAA) is injected at the desired injection position(s) to simulate the distribution of microspheres, and is subsequently imaged using both planar gamma camera imaging (for lung shunt fraction assessment) and SPECT/CT. Pre-treatment 
dosimetry was performed on SPECT/CT using Simplicit ${ }^{90} \mathrm{Y}{ }^{\circledR}$ software (Mirada Medical Ltd, Oxford, UK). Without any contraindications, the patients will proceed to treatment usually within 1-2 weeks. The exact same injection position for ${ }^{99 \mathrm{~m}} \mathrm{Tc}-\mathrm{MAA}$ injection is used for the treatment. Post-treatment ${ }^{90} \mathrm{Y}-\mathrm{PET} / \mathrm{CT}$ is obtained the same day or the day after treatment. Whenever post-treatment dosimetry was performed, it was once again done using Simplicity ${ }^{90} \mathrm{Y}$ software.

\section{${ }^{90} \mathrm{Y}-\mathrm{PET} / \mathrm{CT}$ protocol}

Images were acquired on a Biograph mCT time-of-flight PET/CT scanner with TrueV (Siemens Medical Solutions USA, Inc.). It combines a whole-body LSO PET scanner with a 40 -slice CT scanner. ${ }^{90}$ Y-PET imaging was performed on the treatment day or the day after. Scans were made in two bed positions, with an acquisition time of $15 \mathrm{~min}$. To reconstruct the images, an iterative algorithm (4 iterations, 21 subsets), including a model-based scatter correction method, was used. It encompasses a point spread function model of the detector response together with timeof-flight information. A Gaussian filter of $5 \mathrm{~mm}$ (FWHM) was applied during the post-reconstruction phase. Images were reconstructed on a $200 \times 200$ matrix, and a pixel size of $4 \times 4 \times 3 \mathrm{~mm}^{3}$. To correct for attenuation, low-dose CT with a slice thickness of $3 \mathrm{~mm}$ was acquired with an effective X-ray tube current time product equal to $40 \mathrm{mAs}$ and a $\mathrm{kVp}$ of $100-120 \mathrm{kV}$.

\section{Clinical cases}

\section{Extrahepatic deposition}

\section{Case \#1: ${ }^{90} \mathrm{Y}-\mathrm{PET} / \mathrm{CT}$ to detect excessive lung shunt}

A 65-year-old female patient with a history of alcoholic liver cirrhosis and breast cancer presented with advanced stage hepatocellular carcinoma (HCC) (Barcelona Clinic Liver Cancer C) with a tumor in segment III of the liver and tumor thrombus in the left segmental portal branch (Fig. 1a). She was referred for radioembolization with palliative intent.

Catheterization of the common hepatic artery (CHA) revealed extensive arteriovenous shunting (Fig. 1b). Therefore, a selective injection into the left hepatic artery (LHA) was chosen. Planar scintigraphy showed a considerable lung shunt fraction (LSF) of approximately $50 \%$, contraindicating radioembolization treatment (Fig. 1d). However, planar ${ }^{99 \mathrm{~m}}$ Tc-MAA-scintigraphy is known to overestimate the lung shunt value [4]. Therefore, an additional work-up procedure with a scout dose of holmium-166 $\left({ }^{166} \mathrm{Ho}\right)$ microspheres was performed. ${ }^{166} \mathrm{Ho}$-scout dose (QuiremScout ${ }^{\circledR}$, Quirem
Medical, Deventer, The Netherlands) is the standard workup for ${ }^{166} \mathrm{Ho}-\mathrm{RE}$ and consists of $250 \mathrm{MBq}$ of ${ }^{166} \mathrm{Ho}$-microspheres and has been demonstrated to have a more accurate lung shunt prediction than ${ }^{99 \mathrm{~m}} \mathrm{Tc}-\mathrm{MAA}$ [4]. During ${ }^{166} \mathrm{Ho}-$ scout angiography, a more distal injection location was selected and the scout dose was injected. SPECT/CT showed favorable tumor targeting and a lower lung shunt fraction, quantified as $6 \%$. Taking this into account, we proceeded to perform RE with a conservative activity of $1.3 \mathrm{GBq}$ (aiming for a $120 \mathrm{~Gy}$ average absorbed dose in the target volume). This activity was considered safe, as even with an LSF of $50 \%$, the maximum lung absorbed dose would not exceed $30 \mathrm{~Gy}$.

The next day, ${ }^{90} \mathrm{Y}$-PET/CT showed good tumor targeting, as well as visual signs of a lung shunt (Fig. 1e, f). The activity in the lungs as quantified on ${ }^{90} \mathrm{Y}$-PET/CT revealed an absorbed lung dose of approximately $17 \mathrm{~Gy}$ which was below the assumed threshold of 30 Gy [5]. Three weeks post-treatment, the patient developed symptoms of pneumonia that were unresponsive to antibiotic therapy, there were no anomalies on CECT (Fig. 1g). However, at 3.5 months post-treatment, CECT of the lungs showed changes consistent with radiation-induced fibrosis (Fig. 1h).

${ }^{90} \mathrm{Y}$-PET/CT was used to detect lung shunting and to calculate the lung absorbed dose.

\section{Case \#2: ${ }^{90} \mathrm{Y}-\mathrm{PET} / \mathrm{CT}$ to visualize deposition in the gallbladder}

A 74-year-old male patient with a large unresectable HCC in the right lobe was referred to our institute for radioembolization.

The pre-treatment CECT of the liver showed a close proximity of the tumor to the gallbladder (Fig. 2a) and significant involvement of two parasitic arteries arising from the cystic artery (Fig. 2a, b). After consultation with the patient, the decision was made to include the cystic artery in the treatment and to accept the risk of cholecystitis. The patient was planned for whole liver radioembolization using two injection positions (i.e., right and left hepatic artery).

During work-up angiography, two lobar injection positions were selected and after coil embolization of the gastroduodenal artery, ${ }^{99 \mathrm{~m}} \mathrm{Tc}-\mathrm{MAA}$ was administered. Planar imaging estimated a LSF of 5\% and SPECT/CT showed a favorable distribution of activity in the tumor. Due to the low-spatial resolution of SPECT/CT and the proximity of the tumor to the gallbladder, the exact deposition of activity in the gallbladder could not be determined (Fig. 2c). The patient proceeded for treatment, and glass ${ }^{90} \mathrm{Y}$-microspheres were administered in the LHA (2.4 GBq) and RHA $(4.6 \mathrm{GBq})$ with an aimed average whole liver absorbed dose of $120 \mathrm{~Gy}$. 
Fig. 1 a Contrast-enhanced CT (CECT) of the liver showing hepatocellular carcinoma (HCC) in segment III with tumor thrombus in the left segmental portal branch (arrow). b Digital subtraction angiography (DSA) during work-up angiography demonstrating arteriovenous shunting, note the large systemic collateral vein (arrow). c ${ }^{99 \mathrm{~m}}$ Tc-MAA-SPECT/CT showing good targeting of the tumor and tumor thrombus (arrow). d Planar ${ }^{99 \mathrm{~m}}$ Tc-MAA-scintigraphy displaying lung shunting (LSF $50 \%)$. e, $\mathbf{f}^{90}$ Y-PET maximum intensity projection (MIP) and fusion ${ }^{90} \mathrm{Y}$-PET/CT per-

formed after injection of glass ${ }^{90}$ Y-microspheres in a patient with HCC, demonstrating the presence of activity in the lungs. $\mathbf{g}, \mathbf{h}$ CECT of the chest: $\mathbf{g}$ lungs at 3 weeks post-therapy; $\mathbf{h}$ radiation-induced fibrosis with a typical 'bat-wing' appearance
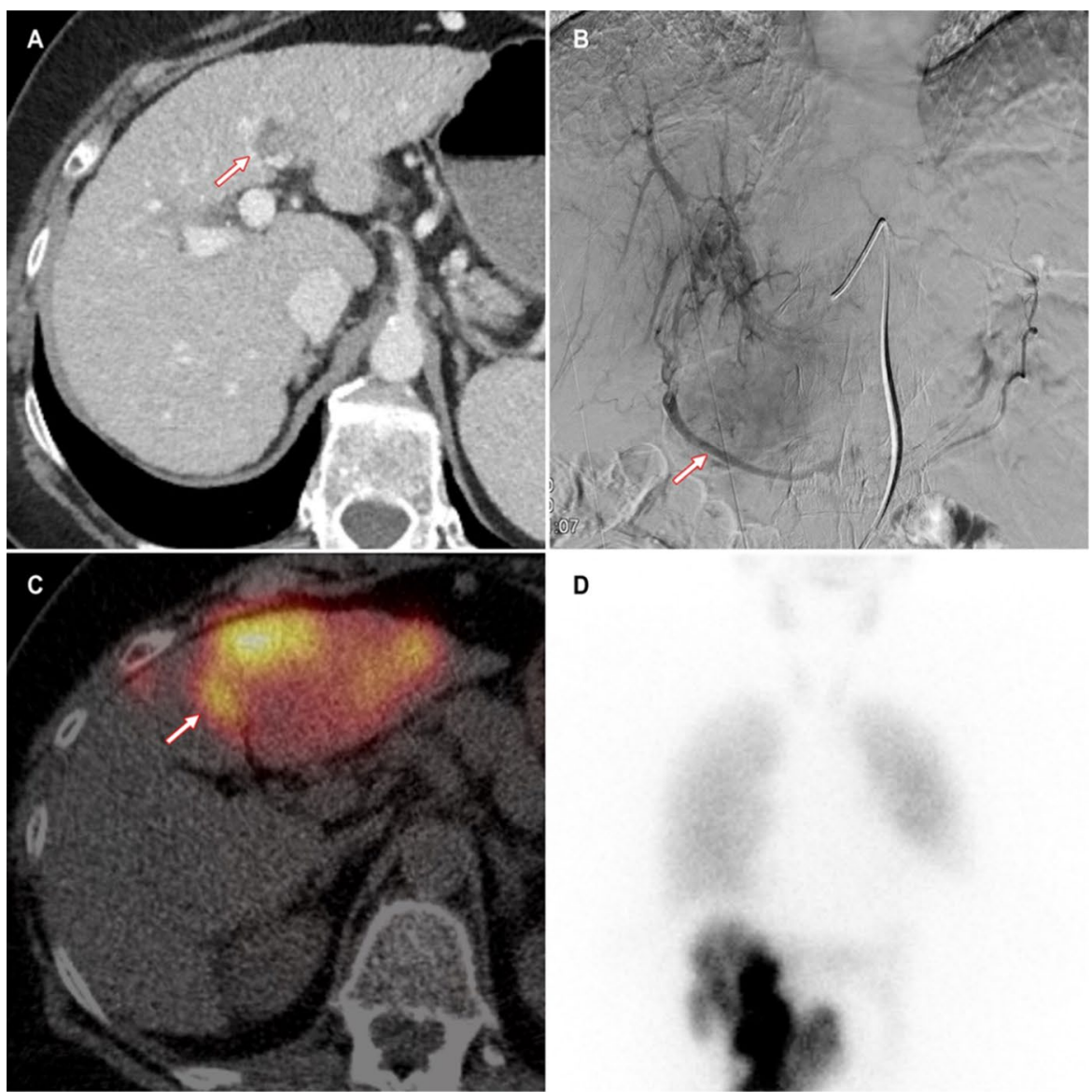

D
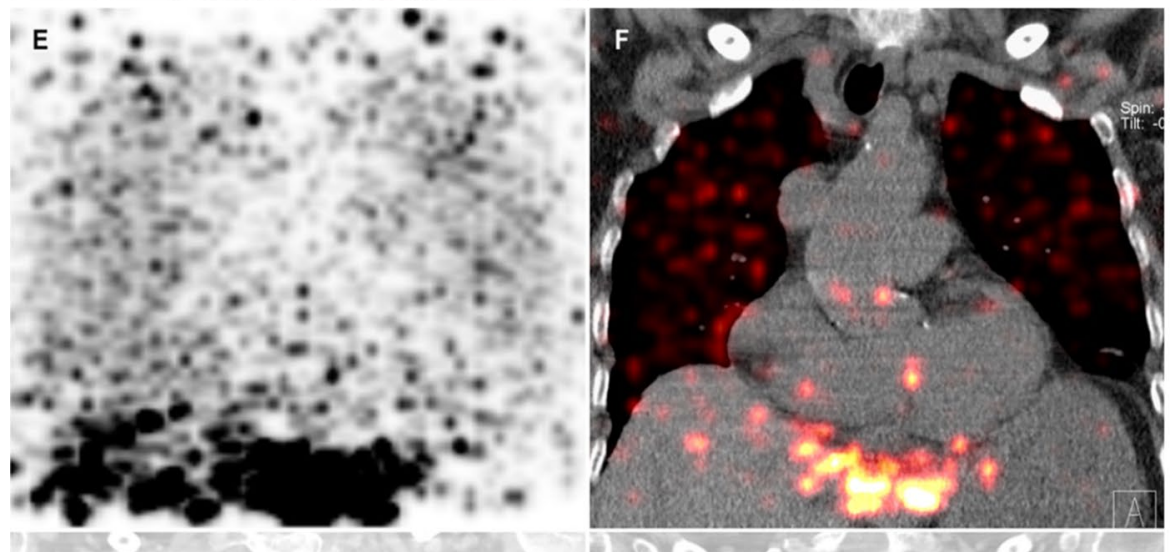

G

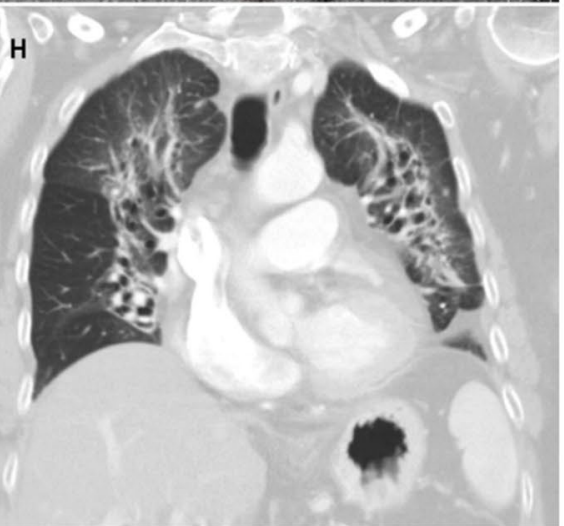


Fig. 2 a CECT of the liver showing HCC involving segments I and IV-VIII. Note the close relationship with the superior gallbladder wall. b Pre-treatment early arterial phase CECT MIP showing the connections to the cystic artery (arrow). c SPECT/CT performed after injection of ${ }^{99 m}$ Tc-MAA, note the difficulty in distinguishing activity in the gallbladder wall. $\mathbf{d}{ }^{90} \mathrm{Y}$-PET/ CT performed after injection of glass ${ }^{90}$ Y-microspheres. The deposition of microspheres in the gallbladder wall is clearly visible. e, f Contrast-enhanced CT with a zoomed view of the gallbladder: e normal aspect of the gallbladder at baseline $\mathbf{f}$ thickening and enhancement of the gallbladder wall at 3 months post-treatment
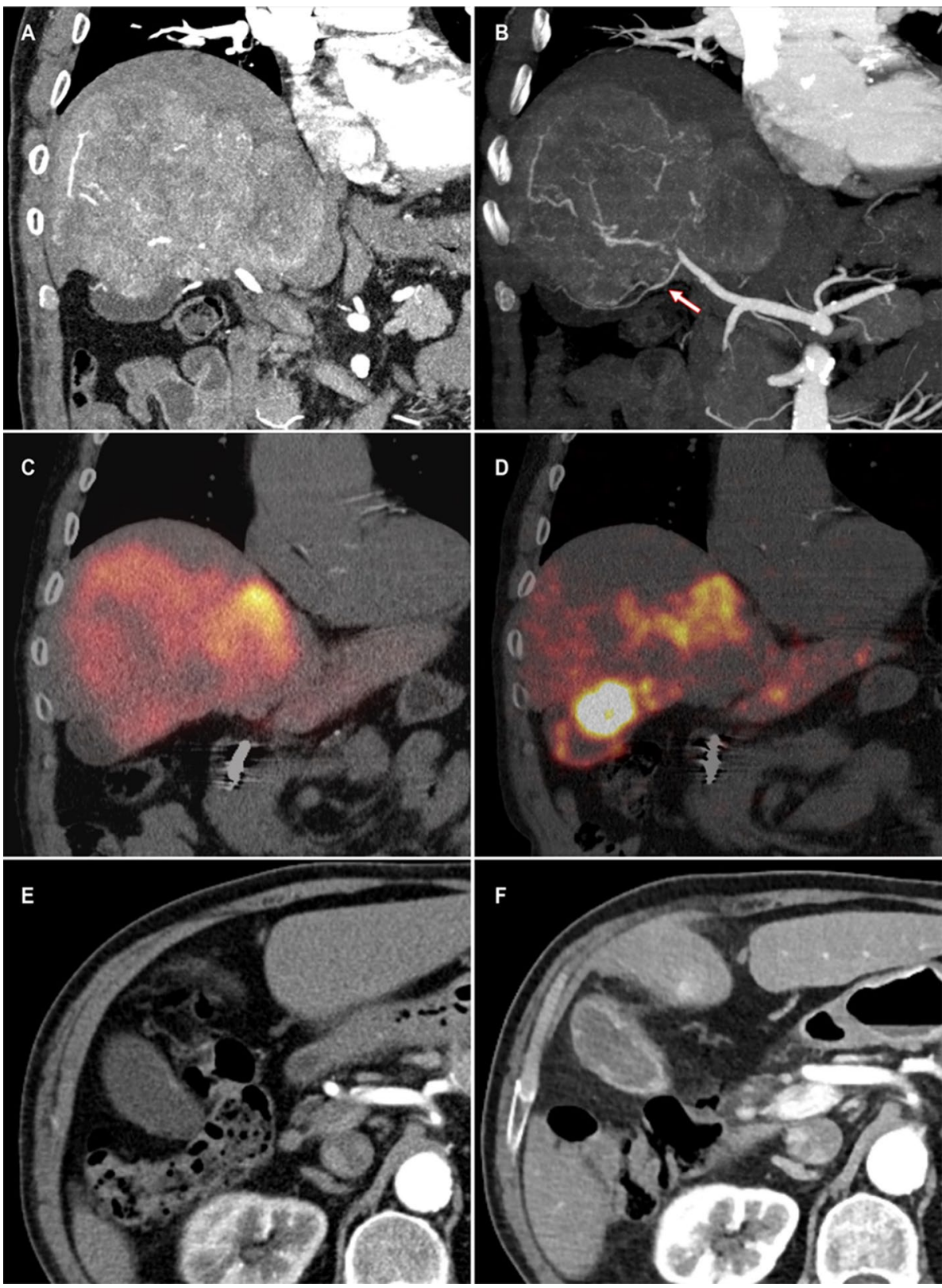

${ }^{90}$ Y-PET/CT was performed the next day, showing a significant amount of activity in the gallbladder (Fig. 2d). There were no symptoms of cholecystitis during follow-up, although CT at 3 months post-treatment showed a thickened gallbladder wall with increased enhancement (Fig. 2f). The aspect of the gallbladder normalized on subsequent followup imaging.

This case illustrated that the amount of activity in the gallbladder may vary between work-up and treatment and that activity in the gallbladder often remains asymptomatic. [6].

\section{Intrahepatic distribution}

\section{Case \#3: ${ }^{90} \mathrm{Y}-\mathrm{PET} / \mathrm{CT}$ to reveal inadvertent selective delivery of ${ }^{90} \mathrm{Y}$-microspheres}

A 68-year-old male with a grade I neuroendocrine tumor of the small bowel and multiple liver metastases (Fig. 3a, b), for which he already received four cycles of intravenous $7400 \mathrm{MBq}$ lutetium-177 $\left({ }^{177} \mathrm{Lu}\right)$-dotatate, was referred for radioembolization after progression of the liver metastases.

The patient was planned for whole liver radioembolization with two injection positions. A suitable injection position was found in the LHA and ${ }^{99 \mathrm{~m}} \mathrm{Tc}-\mathrm{MAA}$ was 
Fig. 3 a, b CECT of the liver showing multiple hypervascular neuroendocrine tumor metastases in the liver. c, $\mathbf{d}$ DSA of the right hepatic artery. c was performed during work-up angiography, note the two segment IV branches (arrows). d was performed during treatment angiography, right before injection of the ${ }^{90} \mathrm{Y}$-microspheres, note the preferential flow toward the second branch (arrow). e, $\mathbf{f}^{99 \mathrm{~m}}$ Tc-MAA-SPECT/CT showing a slightly lower activity distribution in segment IV and relatively homogeneous distribution throughout the rest of liver. $\mathbf{g}, \mathbf{h}$ Post-treatment ${ }^{90} \mathrm{Y}$ PET/CT showing highly intense accumulation of microspheres in segment IV, less intense and homogeneous activity in segments II and III, and almost no activity in segment I and V-VIII
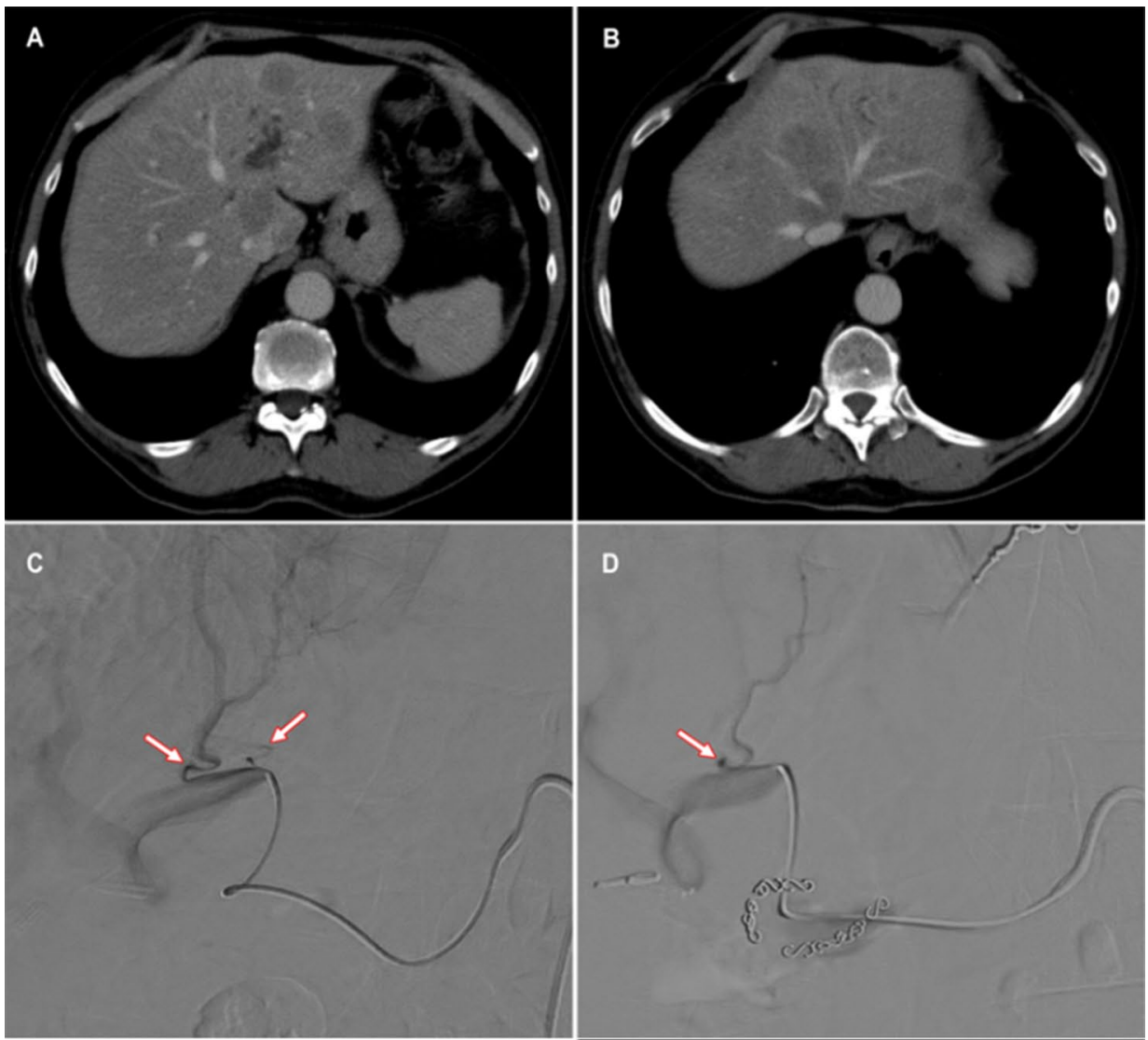

E

D

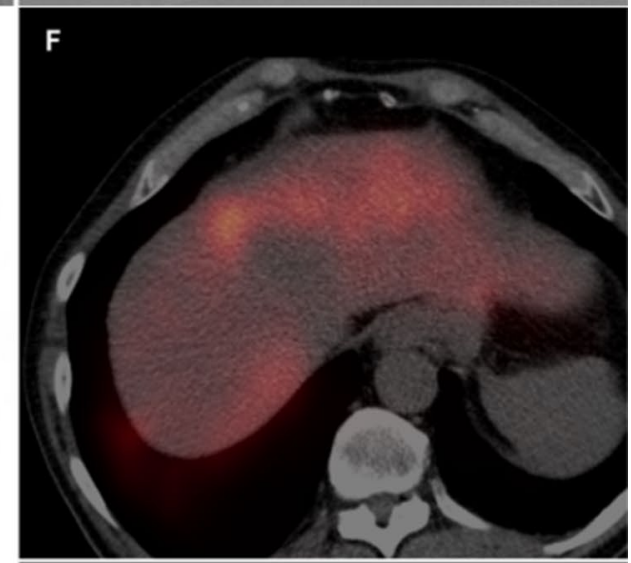

G

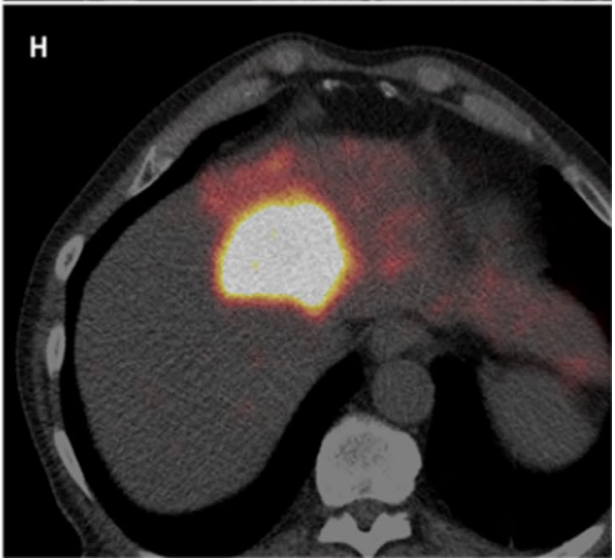


administered. Proximally in the right hepatic artery (RHA), two small medial hepatic arteries were identified, but could not be catheterized separately (Fig. 3c, d). Thus, an injection position proximal to the segment IV branches was selected and ${ }^{99 \mathrm{~m}} \mathrm{Tc}-\mathrm{MAA}$ was administered (Fig. 3c). Planar imaging found a negligible LSF (4.3\%), and SPECT/CT showed good distribution of microspheres throughout the liver, albeit with a slightly lower deposition of activity in segment IV (Fig. 3e, f). After which, the patient proceeded to treatment.

Glass ${ }^{90} \mathrm{Y}$-microspheres were injected into the predetermined positions (LHA activity: $1.1 \mathrm{GBq}$, prescribed average absorbed dose 150 Gy because of high tumor load; RHA activity: $3.5 \mathrm{GBq}$, prescribed average absorbed dose 110 Gy).

${ }^{90} \mathrm{Y}-\mathrm{PET} / \mathrm{CT}$ revealed that almost all activity injected in the RHA had landed in segment IV (Fig. 3g, h). It is hypothesized that this was caused by preferential flow towards the nearby segment IV branch (Fig. 3d) caused by an out-ofcenter placement of the end-hole catheter against the vessel wall. The activity in segment IV was quantified at $3.4 \mathrm{GBq}$ on ${ }^{90} \mathrm{Y}$-PET/CT, which amounts to an absorbed dose of approximately $1400 \mathrm{~Gy}$.

Shortly after treatment, the patient started experiencing severe pain in the liver area and developed a fever. An abscess had formed in the necrotic tissue in segment IV, therefore treatment with wide-spectrum antibiotics was started. Attempts to percutaneously drain the abscess were unsuccessful due to rigidity of the surrounding fibrous tissue and surgical drainage was necessary. In the following weeks, the symptoms improved, only to return 3 days after ceasing antibiotic therapy. Once again, an abscess was found in segment IV, this time the patient underwent a left hepatectomy including the abscess and necrotic tissue. The patient made a full recovery and remains alive to the date of writing this essay.

This case illustrates the essence of ${ }^{90} \mathrm{Y}$-PET/CT to assess whether the treatment was performed as planned. Subtle changes in flow or catheter position may lead to a completely different distribution.

\section{Case \#4: ${ }^{90} \mathrm{Y}-\mathrm{PET} / \mathrm{CT}$ to assess the success of flow redistribution and prediction of REILD}

A 63-year-old male patient with a centrally located cholangiocarcinoma in segment IV and intrahepatic metastases (Fig. 4a) had disease progression after two lines of systemic chemotherapy and was referred for radioembolization.

$\mathrm{CT}$ and work-up angiography showed normal hepatic arterial anatomy and no arteries were coil embolized in this session. ${ }^{99 \mathrm{~m}} \mathrm{Tc}-\mathrm{MAA}$ was injected in the LHA and RHA. The LSF was negligible (3.5\%), and SPECT/CT showed high deposition of activity in the tumor (Fig. 4b).
During treatment angiography, a previously undetected RGA arising from the LHA was visualized and subsequently embolized with microcoils. More distally in the LHA, another gastric vessel was discovered, but could not be catheterized. Thus, the decision was made to (1) move the injection position more distally in the LHA, beyond the ostium of the gastric vessel, and (2) to embolize the tumor feeding segment I branch arising proximally to the new injection position in the LHA to achieve redistribution of blood flow. Glass ${ }^{90} \mathrm{Y}$ microspheres were administered in the LHA (1.2 GBq) and RHA (3.4 GBq), aiming at an average whole liver absorbed dose of $120 \mathrm{~Gy}$, because of the good tumor targeting on ${ }^{99 \mathrm{~m}} \mathrm{Tc}-\mathrm{MAA}$-SPECT/CT.

However, ${ }^{90} \mathrm{Y}$-PET/CT revealed a poor tumor targeting and no redistribution of activity into segment I (Fig. 4c). Post-treatment dosimetry was performed on ${ }^{90}$ Y-PET/CT (Fig. 4e) and revealed that segment I received an absorbed dose of $19.5 \mathrm{~Gy}$, confirming the failure of redistribution. As a result of this, the (whole liver) average normal tissue absorbed dose was $125.3 \mathrm{~Gy}$, while the tumor absorbed dose was only $86.5 \mathrm{~Gy}$. The patient was discharged in a good clinical state.

One month after treatment, the patient complained of increasing pain in the right side of the abdomen and therefore, $\mathrm{CT}$ and ${ }^{18} \mathrm{~F}$-FDG-PET/CT was performed. At 2 months post-treatment, the tumor showed partial metabolic response on ${ }^{18} \mathrm{~F}$-FDG-PET/CT. However, CECT showed a hypodense aspect of the healthy liver, splenomegaly, and ascites. These findings are consistent with radioembolization-induced liver disease (REILD) (Fig. 4g). The patient also developed hepatic encephalopathy, with an increase in serum values of bilirubin had (from 10 to $64 \mu \mathrm{mol} / \mathrm{L}$ ), decrease in albumin (from 42.5 to $29.7 \mathrm{~g} / \mathrm{L}$ ), and increase in the INR (from 1 to 1.23). Four months after treatment, the patient died due to REILD.

In this case, ${ }^{90}$ Y-PET/CT dosimetry showed that the tumor absorbed dose was low, even lower than the healthy liver dose. In addition, ${ }^{90} \mathrm{Y}-\mathrm{PET} / \mathrm{CT}$ demonstrated that the attempt to induce redistribution to segment I had failed. REILD was to be expected based on a 125 Gy healthy liver absorbed dose.

\section{Case \#5: ${ }^{90} \mathrm{Y}$-PET/CT to detect undertreated tumors and to plan retreatment}

A 42-year-old male patient with liver-dominant metastatic colorectal cancer progressed after two lines of systemic chemotherapy. ${ }^{18} \mathrm{~F}$-FDG-PET/CT showed bilobar liver metastases (Fig. 5a, b).

${ }^{99 \mathrm{~m}} \mathrm{Tc}-\mathrm{MAA}$ was injected into three injection positions which were selected during work-up angiography. The first position was proximal in the RHA. The LHA required two injection positions due to an early bifurcation. LSF 
Fig. 4 a CECT of the liver at baseline, large cholangiocarcinoma located in segment IV. b ${ }^{99 \mathrm{~m}}$ Tc-MAA-SPECT/CT showing good tumor targeting and a low amount of activity in the healthy liver tissue, no extrahepatic depositions. c Post-treatment ${ }^{90}$ Y-PET/CT shows no activity in segment I, poor tumor targeting, and high amount of activity in the healthy liver. d CECT of the liver at 2 months post-treatment shows an unchanged tumor size in addition to a heterogeneous and predominantly hypodense aspect of the healthy liver, ascites, and splenomegaly. e Dose map of the liver, highlighting the difference in absorbed dose between the healthy liver, segment I and the central tumor
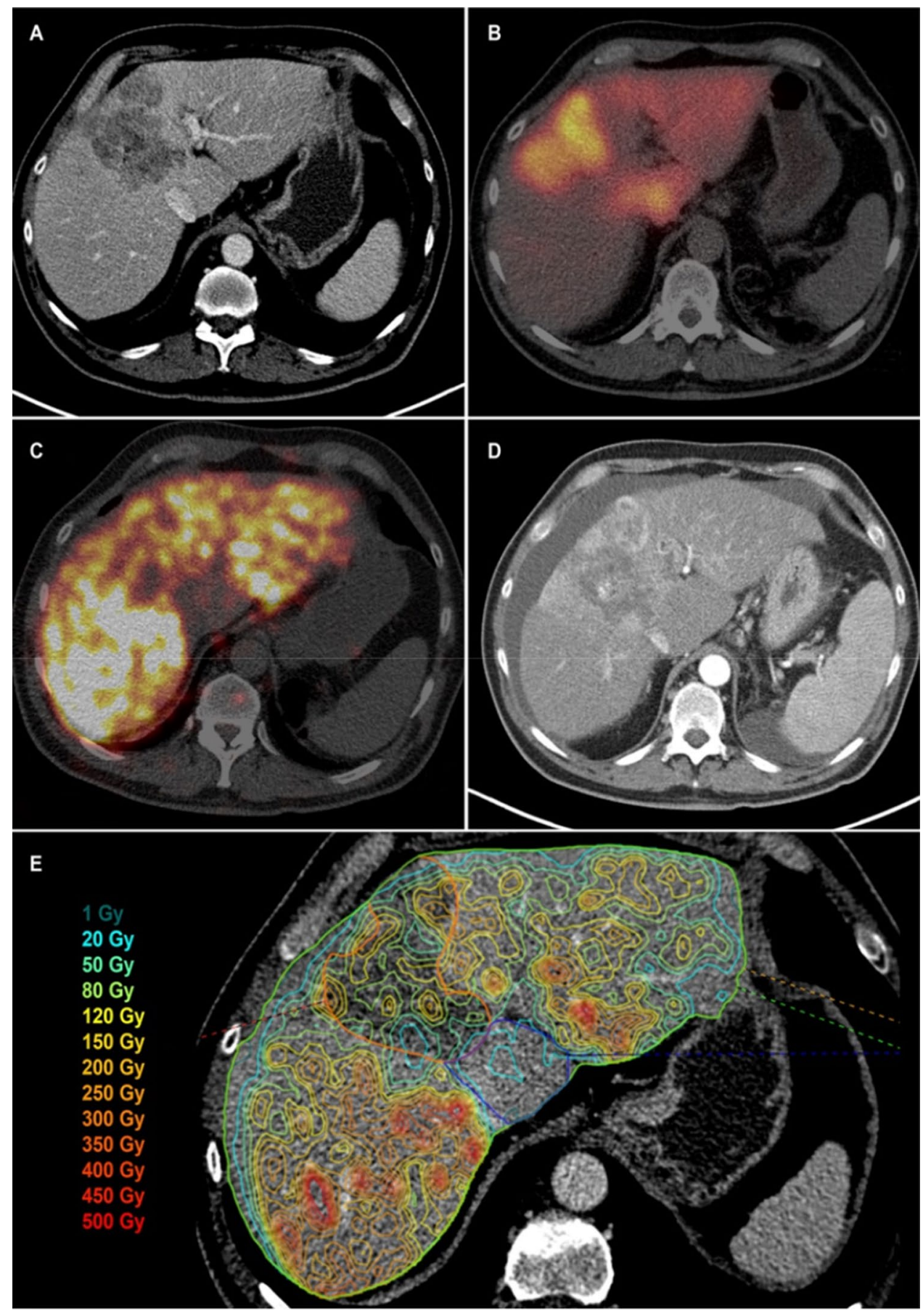

was estimated to be $3 \%$. Segment IV, which contained a large metastasis, showed little to no activity on SPECT/CT (Fig. 5c, d), suggesting that the segmental branch supplying segment IV from the LHA was not included in the proposed injection positions. Therefore, the decision was made to treat the RHA first and plan for treatment of the LHA later.

During the treatment angiography, the overlooked segment IV branch was discovered but could not be catheterized, making selective treatment of the LHA impossible. Therefore, it was decided to convert to a single, central injection position from the CHA, to ensure perfusion of all segments, including segment IV. To prevent reflux into nearby non-target vessels, a Surefire ${ }^{\circledR}$ antireflux catheter (Surefire Infusion System, Surefire Medical Inc., Westminster, CO, USA) was used (Fig. 5f). As literature suggests, a turbulent flow pattern distal of the Surefire catheter makes a homogenous distribution of the microspheres more likely [7]. $2.8 \mathrm{GBq}$ of glass ${ }^{90} \mathrm{Y}$-microspheres that were planned for treatment of the RHA was administered in the CHA. 
Fig. 5 a, b ${ }^{18}$ F-FDG-PET/CT at baseline showing a large colorectal carcinoma liver metastasis between segments IV and VIII. Other metastases are not shown in this image. c, $\mathbf{d}^{99 \mathrm{~m}}$ Tc-MAA-SPECT/CT showing lower deposition of activity in segment IV. e Fusion image of two cone-beam CTs. Contrast injected into the RHA is blue colored, LHA is red. Note the central lesion in segment IV/VIII is vascularized by both hepatic arteries. f Injection of glass ${ }^{90}$ Y-microspheres injection in the CHA via a Surefire(R) antireflux catheter. g, h Post-treatment ${ }^{90}$ Y-PET/ CT showing inhomogeneous deposition of microspheres and preferential flow to the LHA. $\mathbf{i}, \mathbf{j}{ }^{18}$ F-FDG-PET/CT 1 month after therapy shows a clear difference in metabolic response: the left side of the central lesion shows a complete metabolic response, whereas the right side is unchanged
A

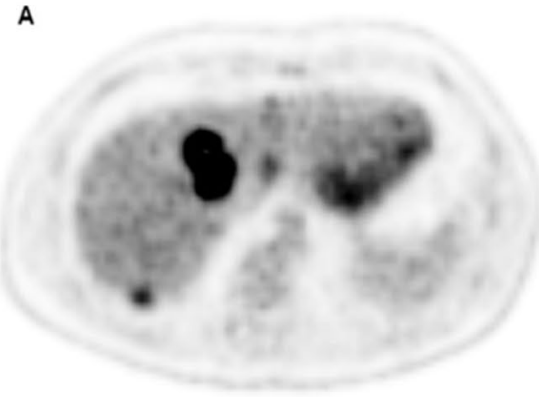

C
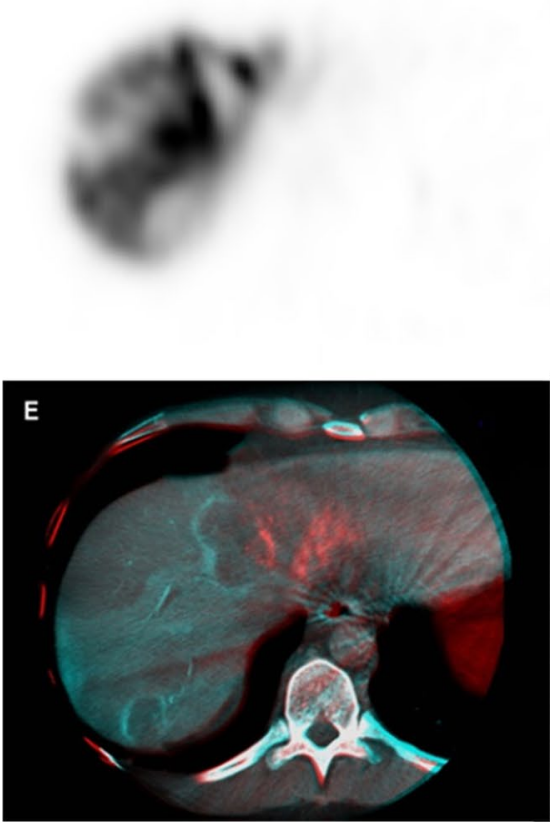

G

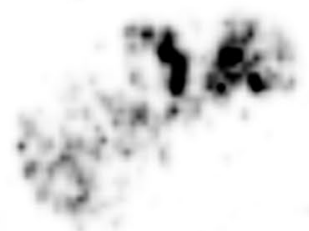

I

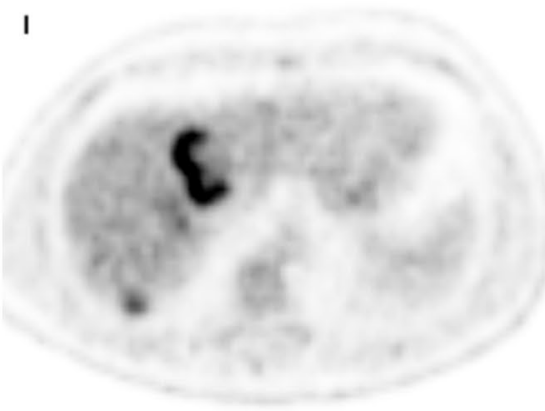

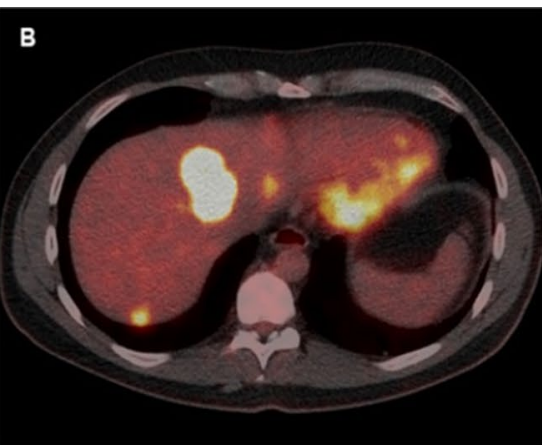

B
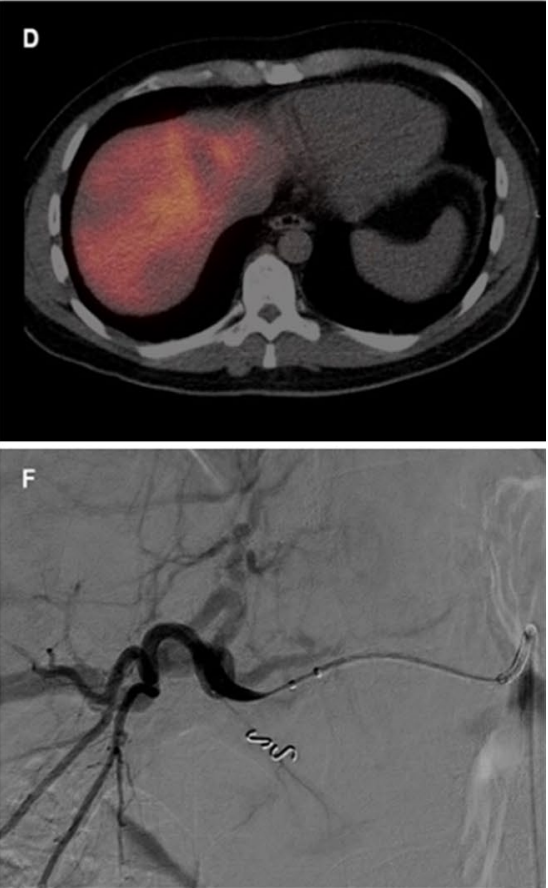

H

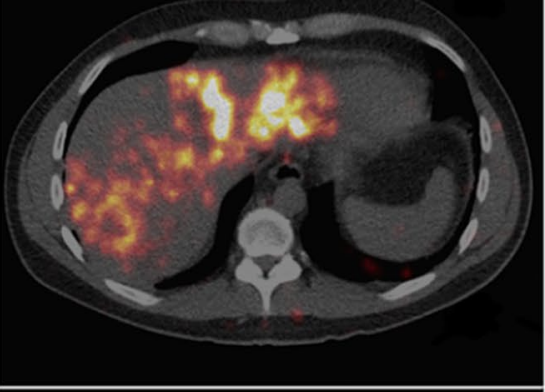

J

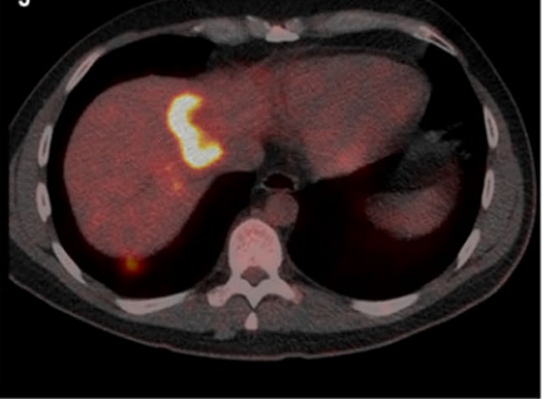


${ }^{90}$ Y-PET/CT showed heterogeneous distribution in the liver with good targeting of tumors in the left lobe and segment IV and significantly lower accumulation in the right liver lobe, caused by preferential flow to the LHA (Fig. 5g, h). Post-treatment dosimetry showed an average absorbed dose of $219 \mathrm{~Gy}$ in the left lobe with a $427 \mathrm{~Gy}$ tumor absorbed dose, whilst the average absorbed dose in the right lobe was 56 Gy with a tumor absorbed dose of $120 \mathrm{~Gy}$. Follow-up ${ }^{18}$ F-FDG-PET/CT at 1 month showed a marked difference in metabolic activity between lesions in the two liver lobes. Tumors supplied by the LHA showed a partial metabolic response, while lesions supplied by the RHA showed unchanged high metabolic activity (Fig. 5i, j). The lesion located in segment IV/VIII, vascularized by both hepatic arteries (as seen on fusion cone-beam CT, Fig. 5e) illustrated this difference with hardly any FDG uptake in the left half of the tumor and unchanged intense FDG uptake in the right half.

An additional radioembolization treatment of the right liver lobe was performed, using ${ }^{90} \mathrm{Y}-\mathrm{PET} / \mathrm{CT}$ dosimetry to determine a safe absorbed dose. Two months later, an additional $1.5 \mathrm{GBq}$ was administered into the RHA, amounting to an additional average absorbed dose of $64 \mathrm{~Gy}$ to the target volume, and a cumulative dose of $120 \mathrm{~Gy}$, which appeared uneventful and safe during follow-up.

In this case, ${ }^{90} \mathrm{Y}$-PET/CT was used for early detection of undertreated tumors and for actively planning for a safe additional absorbed dose for a second treatment.

\section{Discussion}

In this pictorial essay, it was attempted to illustrate the utility of ${ }^{90} \mathrm{Y}$-PET/CT in daily practice. ${ }^{90} \mathrm{Y}$-PET/CT can be used to assess extrahepatic deposition of activity (e.g., lung shunt assessment [Case \#1] and gallbladder [Case \#2]) and intrahepatic distribution of activity (e.g., tumor absorbed dose [Case \#3], healthy liver absorbed dose [Case \#4], and preferential flow [Case \#5]).

Several new insights were gained by performing ${ }^{90} \mathrm{Y}$ PET/CT. In case \#1, the injected activity was tailored as to not exceed the $30 \mathrm{~Gy}$ threshold for lung absorbed dose, and post-treatment imaging confirmed this. However, radiationinduced pneumonitis did occur with a lung absorbed dose of $17 \mathrm{~Gy}$. This led us to rethink our assumptions on this safety threshold. Furthermore, it was shown that redistribution of hepatic blood flow by means of embolizing certain hepatic arteries is not always successful. This method is commonly applied in RE and other interventions such as trans-arterial chemoembolization, to simplify procedures and to reduce risk of complications $[8,9]$. Case \#4 was one of several cases in which this redistribution was not evident on posttreatment imaging.
The presented cases are not a comprehensive account of the utility of ${ }^{90}$ Y-imaging; however, they do make a compelling argument for its implementation.

A commonly raised argument is that post-treatment ${ }^{90} \mathrm{Y}$ PET/CT imaging is not worth the costs and effort, since imaging should only be performed if it leads to relevant clinical consequences. Indeed, once injected, ${ }^{90}$ Y-microspheres distribution cannot be influenced. However, the assessment of the ${ }^{90}$ Y-microsphere distribution may have relevant clinical consequences. It may prove vital to understand and treat complications, and to plan additional treatments if tumors have received an inadequate absorbed dose.

Since recently, there is a European Council Directive $2013 / 59$ [10] that states that all radiation (including radioembolization) to which patients are exposed for therapeutic purpose shall be individually planned and shall have its delivery verified, making individual post-treatment imaging and dosimetry mandatory.

To judge whether a tumor has received a high enough absorbed dose, we must first address what an adequate dose constitutes. Post-treatment ${ }^{90}$ Y-PET/CT-based dosimetric data have been used to successfully demonstrate a dose-response relationship in glass ${ }^{90} \mathrm{Y}$-microsphere radioembolization of hepatocellular carcinoma [11]. In this study, responders had higher median ${ }^{90} \mathrm{Y}$ tumor absorbed doses than non-responders, 225 Gy versus $83 \mathrm{~Gy}$, respectively $(p<0.01)$. All non-responders (stable disease and progressive disease; $n=5 / 27$ ) had tumor absorbed doses lower than $200 \mathrm{~Gy}$, suggesting a possible threshold for treatment efficacy. A prospective study found a dose-response relationship in resin ${ }^{90} \mathrm{Y}$-microsphere radioembolization of colorectal cancer liver metastases [12]. An optimal tumor absorbed dose value for efficacy could not be determined, however, the study did find a minimum value of 40-60 Gy. More research is required to establish more robust absorbed dose thresholds.

To improve the generalizability of dosimetric studies and to allow for inter-site comparability, we must aim to standardize image acquisition and reconstruction protocols. In the QUEST phantom study, an effort was made to compare the quantitative accuracy of ${ }^{90} \mathrm{Y}$ PET-CT imaging for different systems [13]. The study found that current generation PET scanners with time-of-flight were capable of producing comparable ${ }^{90} \mathrm{Y}$ quantifications over a large range of activity and concentration levels. We refer to this study for vendorand model-specific recommendations on reconstructions for quantitative purposes.

In theory, post-treatment ${ }^{90} \mathrm{Y}$-imaging would be redundant if the distribution of microspheres could be accurately predicted by performing ${ }^{99 \mathrm{~m}} \mathrm{Tc}-\mathrm{MAA}$ imaging. Unfortunately, it is known that the microsphere distribution cannot be predicted with $100 \%$ accuracy using the currently available methods. ${ }^{99 \mathrm{~m}} \mathrm{Tc}-\mathrm{MAA}$ has shown poor results in terms of 
predicting the intrahepatic distribution of ${ }^{90} \mathrm{Y}$-microspheres [14]. Wondergem et al. showed that in every procedure, at least one segment had a $>10 \%$ difference in activity between ${ }^{99 \mathrm{~m}} \mathrm{Tc}-\mathrm{MAA}-\mathrm{SPECT} / \mathrm{CT}$ and ${ }^{90} \mathrm{Y}$ SPECT/CT, and $32 \%$ of segments had a difference in activity that was $>30 \%$, underlining the need for post-treatment imaging.

An alternative modality for post-treatment imaging is ${ }^{90} \mathrm{Y}$ bremsstrahlung SPECT. This used to be the only available method for imaging ${ }^{90} \mathrm{Y}$, and is still widely used due to low cost and wide availability [1]. However, it has been demonstrated that ${ }^{90} \mathrm{Y}$-PET is superior to ${ }^{90} \mathrm{Y}$-SPECT in several aspects: higher contrast at similar noise levels, boasting superior image quality, the ability to detect small accumulation of activity, and higher accuracy in making dose estimates than SPECT, which tends to underestimate the activity dose in high-dose regions [3].

In conclusion, PET/CT is an excellent modality for the post-treatment imaging of ${ }^{90} \mathrm{Y}$-microspheres. The presented cases are demonstrations of its ability to predict complications, find undertreated tumors, and aid in dose planning for the retreatment of undertreated tumors. More ${ }^{90} \mathrm{Y}$-PET/ CT-based dosimetric data and research into dose-response and dose-toxicity relationship could lead to improved dose planning and more personalized treatment. Therefore, routine post-treatment ${ }^{90} \mathrm{Y}-\mathrm{PET} / \mathrm{CT}$ is highly recommended.

Authors' contribution M.L. conceived the idea for this essay. A.B., A.A., M.L., and M.S. acquired and reviewed all the cases. The manuscript was drafted by A.A., M.S, and M.B., which was then read and approved by all other authors.

\section{Compliance with ethics standards}

Conflict of interest Our department has received research support from BTG, Terumo and Quirem Medical, it has also received royalties from Terumo and Quirem Medical. Marnix G.E.H. Lam is a consultant for BTG, Terumo and Quirem Medical. Maarten LJ. Smits and Arthur J.A.T. Braat have received compensation for presentations held for Sirtex Medical, Terumo and BTG. Ahmed A. Alsultan and Maarten W. Barentsz declare no conflicts of interest.

Ethical approval All procedures performed in studies involving human participants were in accordance with the ethical standards of the institutional and/or national research committee and with the 1964 Helsinki Declaration and its later amendments or comparable ethical standards.

Informed consent Informed consent was obtained from all individual participants included in studies. All the images presented in this pictorial essay were obtained by the authors at the University Medical Center Utrecht.

Open Access This article is distributed under the terms of the Creative Commons Attribution 4.0 International License (http://creativeco mmons.org/licenses/by/4.0/), which permits unrestricted use, distribution, and reproduction in any medium, provided you give appropriate credit to the original author(s) and the source, provide a link to the Creative Commons license, and indicate if changes were made.

\section{References}

1. Reinders MTM, Mees E, Powerski MJ et al (2018) Radioembolisation in Europe: a survey amongst CIRSE members. Cardiovasc Intervent Radiol 41:1579-1589

2. Braat MNGJA, van Erpecum KJ, Zonnenberg BA et al (2017) Radioembolization-induced liver disease: a systematic review. Eur J Gastroenterol Hepatol 29:144-152

3. Elschot M, Vermolen BJ, Lam MGEH et al (2013) Quantitative Comparison of PET and bremsstrahlung SPECT for imaging the In vivo yttrium-90 microsphere distribution after liver radioembolization. PLoS ONE 8:55742

4. Elschot M, Nijsen JFW, Lam MGEH et al (2014) 99mTc-MAA overestimates the absorbed dose to the lungs in radioembolization: a quantitative evaluation in patients treated with $166 \mathrm{Ho}-$ microspheres. Eur J Nucl Med Mol Imaging 41:1965-1975

5. Narsinh KH, Van Buskirk M, Kennedy AS et al (2017) Hepatopulmonary shunting: a prognostic indicator of survival in patients with metastatic colorectal adenocarcinoma treated with ${ }^{90} \mathrm{Y}$ radioembolization. Radiology 282:281-288

6. Prince JF, Van Den Hoven AF, Van Den Bosch MAAJ et al (2014) Radiation-induced cholecystitis after hepatic radioembolization: do we need to take precautionary measures? J Vasc Interv Radiol 25:1717-1723

7. van den Hoven AF, Prince JF, Bruijnen RCG et al (2016) Surefire infusion system versus standard microcatheter use during holmium-166 radioembolization: study protocol for a randomized controlled trial. Trials 17:520

8. Spreafico C, Morosi C, Maccauro M et al (2015) Intrahepatic flow redistribution in patients treated with radioembolization. Cardiovasc Intervent Radiol 38:322-328

9. Abdelmaksoud MHK, Louie JD, Kothary N et al (2011) Consolidation of hepatic arterial inflow by embolization of variant hepatic arteries in preparation for yttrium-90 radioembolization. J Vasc Interv Radiol 22:1364-1371

10. Chiesa C, Sjogreen Gleisner K, Flux G et al (2017) The conflict between treatment optimization and registration of radiopharmaceuticals with fixed activity posology in oncological nuclear medicine therapy. Eur J Nucl Med Mol Imaging 44:1783-1786

11. Chan KT, Alessio AM, Johnson GE et al (2018) Prospective trial using internal pair-production positron emission tomography to establish the yttrium-90 radioembolization dose required for response of hepatocellular carcinoma. Int J Radiat Oncol 101:358-365

12. van den Hoven AF, Rosenbaum CENM, Elias SG et al (2016) Insights into the dose-response relationship of radioembolization with resin 90Y-microspheres: a prospective cohort study in patients with colorectal cancer liver metastases. J Nucl Med 57:1014-1019

13. Willowson KP, Tapner M, QUEST Investigator Team TQI et al (2015) A multicentre comparison of quantitative (90)Y PET/CT for dosimetric purposes after radioembolization with resin microspheres: the QUEST phantom study. Eur J Nucl Med Mol Imaging 42:1202-1222

14. Wondergem M, Smits MLJ, Elschot M et al (2013) 99mTc-macroaggregated albumin poorly predicts the intrahepatic distribution of $90 \mathrm{Y}$ resin microspheres in hepatic radioembolization. J Nucl Med 54:1294-1301

Publisher's Note Springer Nature remains neutral with regard to jurisdictional claims in published maps and institutional affiliations. 